\title{
PG 1605+072 IN WET XCOV22: SUPPORT FOR THE MULTI SITE SPECTROSCOPIC TELESCOPE
}

\author{
S. L. Schuh ${ }^{1}$, U. Heber ${ }^{2}$, S. Dreizler ${ }^{1}$, S. O'Toole ${ }^{3,2}$, C.S. Jeffery ${ }^{4}$, \\ S. Falter ${ }^{2}$, V.M. Woolf ${ }^{4,5}$, R. L. Riddle ${ }^{6}$, G. Handler ${ }^{7,8}$, Ö. Hürkal, \\ E. Pakštiene ${ }^{9}$, E. W. Klumpe ${ }^{10}$, T. Laurance ${ }^{10}$, M. Vuckovic $^{6}$, S. Zoła ${ }^{11,12}$, \\ S. D. Kawaler ${ }^{6}$, A. Kanaan ${ }^{13}$, H. Monteiro ${ }^{14}$, O. Giovannini ${ }^{15}$, \\ S. O. Kepler ${ }^{16}$, A. Mukadam ${ }^{17}$, J. L. Provencal ${ }^{18}$, A. Nitta ${ }^{19}$, \\ H. Shipman ${ }^{18}$, F. Mullally ${ }^{17}$, A. Grauer ${ }^{20,21}$, M. A. Wood $^{22}$, P. A. Brad- \\ ley $^{23}$, M. Kilic ${ }^{17}$, K. Sekiguchi ${ }^{24}$, R. Crowe ${ }^{25}$, D. J. Sullivan ${ }^{26}$, R. Rosen ${ }^{27}$, \\ C. Clemens $^{27}$, J. Xiaojun ${ }^{28}$, R. Janulis ${ }^{9}$, D. O'Donoghue ${ }^{10}$, W. Ogloza ${ }^{12}$, \\ A. Baran $^{12}$, R. Silvotti ${ }^{29}$, S. Marinoni ${ }^{30}$, G. Vauclair ${ }^{31}$, N. Dolez ${ }^{31}$, \\ M. Chevreton ${ }^{32}$, J. L. Deetjen ${ }^{1}$, T. Nagel ${ }^{1}$, J. M. González Pérez ${ }^{33}$, \\ J.-E. Solheim ${ }^{33}$, R. Østensen ${ }^{34}$, A. Ulla ${ }^{35}$, M. Burleigh ${ }^{36}$, S. Good ${ }^{36}$, \\ T. S. Metcalfe ${ }^{17}$, A. F. M. da Costa $^{16}$, J. E. S. Costa ${ }^{16}$, M. S. O'Brien ${ }^{37}$, \\ S.-L. Kim ${ }^{38}$, H. Lee ${ }^{38}$, A. Sergeev ${ }^{39}$, C. Akan ${ }^{40}$, Ö. Cakirli ${ }^{40}$, M. Paparo ${ }^{41}$ \\ and G. Viraghalmy ${ }^{41}$
}

1 Institut für Astronomie und Astrophysik, Universität Tübingen, Sand 1, D-72076 Tübingen, Germany

2 Dr. Remeis-Sternwarte, Sternwartstrasse 7, D-96049 Bamberg, Germany

3 School of Physics, A28, The University of Sydney, NSW 2006, Australia

4 Armagh Observatory, College Hill, BT61 9DG Armagh, Northern Ireland, United Kingdom

5 Astronomy Department, University of Washington, Box 351580, Seattle WA 98195-1580, U.S.A.

6 Department of Physics and Astronomy, Iowa State University, Ames, Iowa 50011-3160, U.S.A.

7 South African Astronomical Observatory, P.O. Box 9, Observatory 7935, South Africa

8 Institut für Astronomie, Universität Wien, Türkenschanzstraße 17, A1180 Wien, Austria

9 Institute of Theoretical Physics and Astronomy, of Vilnius University, Goštauto 12, LT-2600 Vilnius, Lithuania

10 Department of Physics and Astronomy, P.O. Box 71, 1301 East Main Street, Murfreesboro, TN 37132, U.S.A.

11 Astronomical Observatory, Jagiellonian University, ul. Orla 171, 30244 Crakow, Poland 
12 Mt. Suhora Observatory, Crakow Pedagogical University, ul. Podchorazych 2, 30-084 Cracow, Poland

13 Departamento de Fizika, Universidade Federal de Santa Catarina, Florianopolis, SC - 88040-900, Brazil

14 Instituto Astronomico e Geofisico, USP, Av. Miguel Stefano, 4200, CEP 04301-904 Sao Paulo, SP, Brazil

15 Departamento de Física e Quimica, Universidade de Caxias do Sul, 95001-970, Caxias do Sul, RS, Brazil

16 Universidade Federal do Rio Grande Do Sul, Brazil, Instituto de Fisica, Caixa Postal 15051, 91501-970 Porto Alegre RS, Brasil

17 University of Texas at Austin, 17.310 RLM Hall, Austin, TX-78712, U.S.A.

18 Department of Physics and Astronomy, University of Delaware, Newark, DE 19717-2570, U.S.A.

19 Apache Point Observatory, 2001 Apache Point Road, P.O. Box 59, Sunspot, NM 88349-0059, U.S.A.

20 Department of Physics and Astronomy, University of Arkansas at Little Rock, 2801 S. University Avenue, Little Rock, AR 72204-1099, U.S.A.

21 Department of Astronomy / Steward Observatory, University of Arizona, $933 \mathrm{~N}$ Cherry Ave., Tucson, AZ 85721-0065, U.S.A.

22 Department of Physics and Space Sciences at SARA Observatory, Florida Institute of Technology, 150 West University Bouleward, Melbourne, FL 32901-6975, U.S.A.

23 Los Alamos National Laboratory, P.O. Box 1663, Los Alamos, NM 87545, U.S.A.

24 Subaru Telescope, National Astronomical Observatory of Japan, 650 North A'ohoku Place, Hilo, Hawaii 96720, U.S.A.

25 Institute for Astronomy at the University of Hawaii, 640 North Aohoku Place, Kukahauula, Hilo 132, Hawaii 96720, U.S.A.

26 School of Chemical and Physical Sciences, Victoria University of Wellington, P.O. Box 600, Wellington, New Zealand

27 Department of Physics and Astronomy, University of North Carolina, Phillips Hall, CB \#3255, Chapel Hill, NC 27599-3255, U.S.A.

28 Beijing Astronomical Observatory, National Astronomical Observatories, Chinese Academy of Sciences, 20A Datun Road, Chaoyang District, Beijing, China

29 Osservatorio Astronomico di Capodimonte, via Moiarello 16, 80131, Napoli, Italy

30 Dipartimento di Astronomia, Università di Bologna, Via Ranzani 1, 40126 Bologna, Italy

31 Observatoire Midi-Pyrénées, 14 avenue Edouard Belin, F-31400 Toulouse, France

32 Observatoire Paris-Meudon, Place Jules Janssen, F-92195 Meudon, France

33 Institutt for Fyzikk, Universitet $i$ Tromsø, N-9037 Tromsø, Norway

34 Isaac Newton Group, Apartado de correos 321, E-38700 Santa Cruz de la Palma, Tenerife, Spain

35 Universidade de Vigo, Departamento de Física Aplicada, Facultade de Ciencias, Campus Marcosende-Lagoes, E-36200 Vigo (Pontavedra), Spain 
36 Department of Physics and Astronomy, University of Leicester, University Road, Leicester LE1 7RH, United Kingdom

37 Department of Astronomy, Yale University, 260 Whitney Avenue, New Haven, CT 06511, U.S.A.

38 Korea Astronomy Observatory, San 61-1, Whaam-Dong, Youseong-Gu, Taejeon, R. of Korea 305-348, Korea

39 Main Astronomical Observatory, National Academy of Sciences of Ukraine, 27 Akademika Zabolotnoho St., 03680 Kyiv, Ukraine

40 Ege University Observatory, Science Faculty, Department of Astronomy and Space Sciences, Bornova 35100, Izmir, Turkey

41 Konkoly Observatory, Box 67, H-1525 Budapest XII, Hungary

Received September 20, 2002

Abstract. The Multi-site spectroscopic telescope is a virtual instrument and the name of a collaboration that opens up a new observational window by combining continuous observations of spectroscopic variations and simultaneous photometric monitoring. This constitutes an enormous observational effort, but in return promises to finally provide access to a mode identification for and an asteroseismological analysis of the pulsating sdB star PG 1605+072. Multi-Site Spectroscopic Telescope observations for this object have been secured during a large coordinated campaign in May and June of the year 2002. The frequency resolution and coverage of the photometric time series has been noticeably enhanced by a significant contribution from the Whole Earth Telescope, which was used to observe PG $1605+072$ as an alternate target during the WET XCov22 campaign, also conducted in May 2002. This paper briefly outlines the motivation for the MSST project and tries to give a first assessment of the overall quality of the data obtained, with a focus on the Whole Earth Telescope observations.

Key words: stars: interiors - stars: individual: PG 1605+072

\section{MOTIVATION}

\subsection{Pulsating sdB stars}

Subdwarf B stars occupy a region in the Hertzsprung-Russell diagram that standard tracks of stellar evolution seem to carefully avoid. Although several possible scenarios to form sdB stars have been proposed, most of them involving binary evolution, the problem is still far from solved. Therefore, when a fraction of the sdB stars was discovered to be variable, the prospect of being able to use asteroseismology in addition to spectral analysis to contribute to the knowledge about the origins of these exotic objects immediately triggered both efforts to increase the number of known pulsators as well as first attempts towards an asteroseismological analysis of selected objects. Since pulsating stars allow a direct investigation of 
their structure, the hope is that the evolutionary history of sdBs can be constrained from the evaluation of their pulsation modes. A recent review of the current status in this field was given by Charpinet (2001).

But while emphasizing the great potential of asteroseismology with sdB variables (sdBV), that article also clearly demonstrates again that the process of mode identification, necessarily required for an asteroseismological study, is a difficult task and is even more complicated for sdBV stars than for objects such as pulsating white dwarfs. While the latter tend to show a nearly equidistant mode pattern for consecutive overtones, no such relation that could be taken advantage of for mode identification is evident for sdBV stars. So obviously, observational methods beyond purely photometric ones are needed to further progress the interpretation of the sdBVs' pulsational behaviour.

Recent feasibility studies for time resolved multi-channel photometry and spectroscopy have shown that such approaches can successfully identify the wavelength dependency of pulsation amplitudes and measure radial velocity variations. Since, for a full resolution of the frequency spectra associated with these values, long-term uninterrupted measurements are required for multi-periodic objects (which are the interesting ones in terms of asteroseismological potential), these individual efforts have eventually culminated in the establishment of the Multi-Site Spectroscopic Telescope. PG 1605+072, an object which has also been used in several of the feasibility studies, was deemed to be the best suited pulsating sdB star to be investigated by such a coordinated campaign.

A concise description of the motivation for the Multi-Site Spectroscopic Telescope, an overview of all observations secured in the May 2002 campaign, and its ultimate scientific goals can be found in a separate article by Heber et al. (2003). The purpose of this article is to describe the additional photometric observations contributed to the overall observational effort by the Whole Earth Telescope.

\section{2. $P G 1605+072$}

PG 1605+072 was first discovered in the Palomar-Green Catalog of Ultraviolet Excess Stellar Objects (Green et al. 1986), and appears in the catalogue of spectroscopically identified hot subdwarf stars by Kilkenny et al. (1988). In 1998, Koen et al. reported that it belongs to the group of EC 14026 stars, the designation for sdB variables derived from the prototype of this class. With this discovery of the variability of PG $1605+072$, it was not only immediately 
evident that this bright star is pulsating in more than just a handful of modes, a rarity in sdBVs, but also that its pulsations are both larger and of longer period than in other objects of this kind. Under such favourable conditions, and driven by the question of what differences in the evolutionary status may account for the differences in the observed pulsational behaviour, the realisation of a multi-site photometric campaign was the logical next step.

The analysis of that campaign by Kilkenny et al. (1999) confirmed that, with $\sim 50$ individual frequencies detected, PG 1605+072 is extraordinarily rich in modes. At $m_{V}=12.8$, with a pulsation amplitude of $0.027 \mathrm{mi}^{1}$ at the main period of $482 \mathrm{~s}, \mathrm{PG} 1605+072$ was also one of the obvious choices when it became clear that, despite the wealth of photometric observations available, observations of a different kind would probably be necessary to achieve mode identification for sdBVs. While Kilkenny et al. did present a comparison of the pulsational behaviour of PG $1605+072$ with a stellar model, they refrained from assigning individual modes in the star to those of their particular model.

Before coming back to the extension of observations to colour and spectroscopic time series, this paragraph compiles a few more characteristics for PG $1605+072$. According to the determination by Heber et al. (1999) on the basis of a time-averaged spectrum, the atmospheric parameters are $T_{\text {eff }}=32300 \mathrm{~K}, \log g=5.25$ and $\log n_{\mathrm{He}} / n_{\mathrm{H}}=-2.53$, and the star rotates rather rapidly at $v \sin i=$ $39 \mathrm{~km} \mathrm{~s}^{-1}$. This rotation constitutes both a valuable aid with mode identification by lifting the m-degeneracy, as well as a further complication due to potential re-coupling effects of the rotation to the pulsations themselves. Apart from these additional difficulties, an asteroseismological analyis also is at risk to be confused on a more fundamental level: given the high number of frequencies observed in PG $1605+072$, even a time base of the order of weeks may be at the limit of yielding the frequency resolution required to separate them all. Furthermore, if amplitude modulation occured on similar time scales, and there are indications that this may be happening, the frequency pattern could in the worst case not be fully resolved before it has changed again.

Despite these potential obstacles in the analysis, several series of time-resolved spectroscopic observations of PG 1605+072 were obtained following the example of Jeffery and Pollaco (2000) who first undertook attempts to apply this technique to sdB stars. Measure-

\footnotetext{
${ }^{1}$ mi: modulation intensity, cf. for example Kilkenny et al. (1999)
} 
ments of radial velocity variations in PG $1605+072$ have been reported in O'Toole et al. (2000, 2002), Woolf et al. (2002), Falter (2001) and Falter et al. (2003). Woolf et al. and Falter additionally comment on line profile variations.

These individual spectroscopic observations (in the case of Falter et al. backed by simultaneous multi-colour photometry) all suffer from the limited frequency resolution and aliasing problem encountered with single-site observations. On the observational side, the data gathered during the Multi-Site Spectroscopic Telescope campaign (see next Section) constitutes a huge improvement over these earlier efforts. Its usefulness however depends in exactly the same way on the availability of good models for an interpretation of the data.

From both spectral analysis and the length of the pulsation periods, it follows that PG $1605+072$ has a relatively low gravity and consequently its luminosity is considerably larger than predicted for an EHB star. Therefore, PG $1605+072$ probably has evolved off the extreme horizontal branch already. The large pulsational amplitudes are also consistent with the location in the part of the instability region occupied by the post-EHB stage. A stellar evolution model and an investigation of its pulsational properties specifically for PG 1605+072 has been presented by Kawaler (1999), while Falter (2001) has brought forward a pulsational model for this object that attempts a detailed mapping of the wavelength dependent light variations across the stellar surface.

Table 1. Overall data in 2002; ff - filling factor.

\begin{tabular}{lrllll}
\hline Type of observations & & From & To & {$[\mathrm{h}]$} & $\mathrm{ff}$ \\
\hline high-resolution spectroscopy & total & $05 / 14$ & $05 / 29$ & 27 & $7 \%$ \\
\cline { 3 - 6 } & total & $05 / 19$ & $06 / 26$ & 152 & $16 \%$ \\
medium-resolution spectroscopy & & & & \\
during main campaign only & $05 / 19$ & $05 / 28$ & 58 & $22 \%$ \\
second part only & $06 / 14$ & $06 / 26$ & 94 & $30 \%$ \\
\cline { 2 - 5 } & total & $05 / 05$ & $06 / 04$ & 328 & $44 \%$ \\
photometry & WET & $05 / 05$ & $05 / 21$ & 127 & $31 \%$ \\
MSST & $05 / 14$ & $06 / 04$ & 272 & $51 \%$ \\
multi-color & $05 / 19$ & $05 / 22$ & 21 & $22 \%$ \\
\hline
\end{tabular}




\section{THE MULTI-SITE SPECTROSCOPIC TELESCOPE}

\subsection{The overall campaign}

Since the sucessful realisation of the Multi-Site Spectroscopic Telescope campaign, PG 1605+072 can probably be claimed to be the best-observed EC 14026 star. Taking the MSST and WET collaborations together, the campaign meant that several dozen astronomers concerted their efforts in the period between May and June 2002 to observe one single star for a total of nearly 500 hours. These observations fall into three categories. The first represents the central idea of MSST to collect high-resolution $(\sim 1 \AA)$ spectroscopy, presumably useful for both radial velocity measurements as well as detailed line profile variation analysis including equivalent width variation measurements. The second consists of mediumresolution $(\sim 3 \AA)$ spectroscopic data with the purpose of obtaining radial velocity measurements on a longer time base than would have been possible with the larger telescopes alone. The third category compiles extensive photometric observations with the aim to fully resolve the frequency spectrum and detect as many modes as possible, to serve as a reference for the less well resolved temporal spectra of the simultaneously acquired spectroscopic observations, and to allow a comparison of the amplitude ratios from light and radial velocity variations.

Table 1 gives an overview of the data obtained for the individual types of observations, and further details the origin of the contributions to the photometry (since some of the data overlap, the sum total is smaller than the sum of the contributions). In addition to the time series photometric data in one passband, a small subset of the photometric data has been taken in four different passbands simultaneously. Using such data, Falter et al. (2003) have successfully demonstrated that the pulsation amplitudes in the different passbands show a wavelength dependency.

\subsection{Photometry from the Whole Earth Telescope}

The Whole Earth Telescope campaign XCov22 was shared between the primary target PG 1456+103 (PI G. Handler) and the secondary target PG 1159-035 (PI S. O'Brien). Due to a large number of participating observatories and generally favourable conditions, the first of the two alternate targets PG $1605+072$ and Feige 48 could be amazingly well covered by WET during the main campaign, perhaps assisted by its easy accessibility with small telescopes due to its greater brightness than the main targets. More than a third of 
the data that will go into the pool of photometric observations to be analysed for MSST will come from WET observations.

The full list of participants, all participating observatories and more details can be found on the XCov22 campaign web pages at http://wet.iitap.iastate.edu/XCov22/. In Table 2, we only list the observatories and the observers that have contributed data on PG 1605+072, mainly to serve as a key to the observatory abbreviations used in Table 3.

Table 2. Contributing observatories.

\begin{tabular}{lllll}
\hline Observatory & Telescope & Instrument & Location & Observers* $^{*}$ \\
\hline CTIO & $1.5 \mathrm{~m}$ & PMT & Chile & AK, HM \\
McDonald & $2.1 \mathrm{~m}$ & CCD & United States & AM, JP, HS, FM \\
Mt. Bigelow & $1.5 \mathrm{~m}$ & PMT & United States & AG \\
SARA & $1.0 \mathrm{~m}$ & CCD & United States & MW \\
Mauna Kea & $0.6 \mathrm{~m}$ & PMT & Hawaii, USA & PB, MK \\
SSO & $1.0 \mathrm{~m}$ & CCD & Australia & DS, RR \\
Bohyunsan & $1.8 \mathrm{~m}$ & CCD & Korea & SLK \\
Terskol & $2.0 \mathrm{~m}$ & CCD & Ukraine & AS \\
SAAO & $1.9 \mathrm{~m}$ & PMT & South Africa & DOD \\
Mt. Suhora & $0.6 \mathrm{~m}$ & PMT & Poland & WO, AB \\
Piszkestetö & $1.0 \mathrm{~m}$ & CCD & Hungary & MP, GV \\
Loiano & $1.5 \mathrm{~m}$ & PMT & Italy & RS, SM \\
Tenerife & $0.8 \mathrm{~m}$ & $3 \mathrm{ch}$ PMT & Tenerife, Spain & JMG, JES \\
JKT & $1.0 \mathrm{~m}$ & CCD & La Palma, Spain MB, SG \\
Itajuba & $1.6 \mathrm{~m}$ & 2ch, CCD & Brazil & ADC, JESC \\
\hline
\end{tabular}

*AK: A. Kanaan, HM: H. Monteiro, AM: A. Mukadam, JP: J. Provencal, HS: H. Shipman, FM: F. Mullally, AG: A. Grauer, MW: M. Wood, PB: P. Bradley, MK: M. Kilic, DS: D. Sullivan, RR: R. Rosen, SLK: S.-L. Kim, AS: A. Sergeev, DOD: D. O'Donoghue, WO: W. Ogloza, AB: A. Baran, MP: M. Paparo, GV: G. Viraghalmy, RS: R. Silvotti, SM: S. Marinoni, JMG: J.M. Gonzalez Perez, JES: J.-E. Solheim, MB: M. Burleigh, SG: S. Good, ADC: A.F.M. da Costa, JESC: J.E.S. Costa

\section{OBSERVATIONS}

\subsection{Whole Earth Telescope data}

A final data reduction still has to be done. All of the data shown in this paper comes directly from the preliminary data reduction performed during the campaign at WET headquarter (Riddle, these proceedings). We note that for all data sets the object counts have been sky background corrected, some have been corrected for trends using one or several reference stars, all have been corrected for extinction effects or other low-frequency variations using low- 
order polynomials when necessary, and bad data points have been removed. The individual data sets were normalized to remove the possible effects of different instrumental set-ups, and the mean value of unity was subtracted so that the flux is eventually given in modulation intensity units. The times denote the barycentrically corrected middle of each exposure. The preliminary light curve just described was compiled from the data sets listed in Table 3. For the data set lna-023, it was necessary to re-adjust the flux level since the mean value of that part of the light curve was not zero but minus one. After the appropriate correction, it nicely fits with the other data.

Figure 1 gives an overview of the total Whole Earth Telescope XCov22 data of PG 1605+072. The actual data are plotted, and the pulsations as well as effects of amplitude modulation due to frequency beating are clearly visible. The good coverage is also evident, although the regular complete lack of data for two hours each day between 19.5 and $21.5 \mathrm{~h} \mathrm{UT}$ will still lead to daily aliases in the window function.

\subsection{Quality of the data}

It is clear that, on the basis of a preliminary data reduction as used here, one can only comment on a few limited aspects of the data. In the remainder of this text, the following facets will be highlighted: agreement between overlapping data, the window functions for the full and divided WET data, and its frequency resolution.

A good possibility to evaluate both the timing accuracy as well as the run of the flux is to compare overlapping data sets. Within the WET data, a total of 12 pairs of data sets partly overlap; for seven of these, the overlap is longer than one hour (all other overlaps have a duration of less than half an hour, see Table 4). Only the pairs of data with longer overlaps were considered for Fig. 2; since one pair additionally overlaps with a third data set, these three data sets are all shown together. The first quality test deals with the time stamps. To determine shifts in the overlapping data sets with respect to each other, the data sets were interpolated onto a common time grid, and the cross-correlation was calculated for a series of offsets. The maximum of the cross-correlation function was then fitted with a Gaussian to find the exact deviation from zero for the highest peak. Whenever this value was considerable larger than $1 \mathrm{~s}$ (the accuracy limit imposed by the interpolation and fitting processes), this offset was used to shift the light curves used to produce Fig. 2. 
Table 3. WET XCov22 data sets on PG 1605+072.

\begin{tabular}{|c|c|c|c|c|}
\hline Run ID & Observatory & UT date & Start & Length [h] \\
\hline suh-106 & Mt. Suhora & 5 May 02 & $01: 27-01: 56$ & 0.4 \\
\hline suh-107 & Mt. Suhora & 5 May 02 & $22: 35-02: 05$ & 3.5 \\
\hline sara $140-5$ & SARA & 6 May 02 & $08: 30-11: 43$ & 3.3 \\
\hline suh-108 & Mt. Suhora & 6 May 02 & $21: 20-01: 48$ & 4.4 \\
\hline ter02 & Terskol & 7 Mav 02 & $00: 24-00: 58$ & 0.6 \\
\hline ra381 & CTIO & 7 May 02 & $07: 34-10: 01$ & 2.6 \\
\hline adg-515 & Mt.Bigelow & 7 May 02 & $09: 42-11: 11$ & 1.5 \\
\hline ra384 & CTIO & 8 May 02 & $05: 26-09: 58$ & 4.4 \\
\hline korea001 & Bohyunsan & 8 May 02 & $13: 02-18: 28$ & 5.4 \\
\hline ra386 & CTIO & 9 May 02 & $05: 57-10: 00$ & 4.0 \\
\hline $\operatorname{dod} 059$ & $\mathrm{SAAO}$ & 10 May 02 & $03: 14-03: 59$ & 0.7 \\
\hline jes0202 & Tenerife & 10 May 02 & $03: 46-05: 16$ & 1.5 \\
\hline ra388 & CTIO & 10 May 02 & $06: 24-09: 53$ & 3.5 \\
\hline B0017 & $\mathrm{SSO}$ & 10 May 02 & $17: 43-19: 18$ & 1.6 \\
\hline jes0204 & Tenerife & 11 May 02 & $02: 16-03: 51$ & 1.6 \\
\hline jes0205 & Tenerife & 11 May 02 & $04: 38-05: 20$ & 0.7 \\
\hline ra390 & CTIO & 11 May 02 & $05: 51-09: 56$ & 4.1 \\
\hline pab-0195 & Mauna Kea & 11 May 02 & $11: 56-14: 40$ & 2.8 \\
\hline B0021 & SSO & 11 May 02 & $17: 35-19: 24$ & 1.8 \\
\hline korea006 & Bohyunsan & 11 May 02 & $17: 54-19: 27$ & 1.6 \\
\hline jes0207 & Tenerife & 12 May 02 & $02: 52-05: 08$ & 2.3 \\
\hline ra393 & CTIO & 12 May 02 & $05: 43-09: 46$ & 4.0 \\
\hline lna-006 & Itajuba & 12 May 02 & 06:03 - 08:02 & 2.0 \\
\hline B0025 & $\mathrm{SSO}$ & 12 May 02 & $18: 19-19: 09$ & 0.9 \\
\hline jes0208 & Tenerife & 12 May 02 & $21: 50-05: 15$ & 7.4 \\
\hline lna-008 & Itajuba & 13 May 02 & $02: 59-07: 05$ & 4.9 \\
\hline jkt-002 & JKT & 13 May 02 & $23: 18-00: 42$ & 1.4 \\
\hline $\mathrm{mp}-001$ & Piszkestetö & 13 May 02 & $23: 23-01: 45$ & 2.4 \\
\hline loi-002a & Loiano & 14 May 02 & $00: 26-00: 55$ & 0.5 \\
\hline loi-002b & Loiano & 14 May 02 & $01: 09-01: 43$ & 0.4 \\
\hline sara143-5 & SARA & 14 May 02 & $07: 35-11: 41$ & 4.1 \\
\hline B0030 & SSO & 14 May 02 & $18: 11-19: 11$ & 1.0 \\
\hline loi-004 & Loiano & 14 May 02 & $23: 30-01: 51$ & 2.3 \\
\hline jkt-004 & JKT & 15 May 02 & 00:04 - 04:07 & 4.1 \\
\hline jgp0211 & Tenerife & 16 May 02 & $01: 32-04: 59$ & 3.5 \\
\hline lna-016 & Itajuba & 16 May 02 & $05: 25-07: 54$ & 2.5 \\
\hline $\mathrm{a} 0236$ & McDonald & 16 May 02 & $09: 02-10: 58$ & 1.9 \\
\hline pab-0197 & Mauna Kea & 16 May 02 & $10: 49-14: 35$ & 3.8 \\
\hline jgp0213 & Tenerife & 17 May 02 & $01: 52-04: 59$ & 3.1 \\
\hline $\ln a-018$ & Itajuba & 17 May 02 & $05: 20-07: 49$ & 2.5 \\
\hline a0238 & McDonald & 17 May 02 & $07: 42-11: 11$ & 3.5 \\
\hline loi-005 & Loiano & 17 May 02 & $22: 51-01: 59$ & 3.1 \\
\hline jgp0215 & Tenerife & 18 May 02 & $01: 51-05: 01$ & 3.2 \\
\hline $\ln a-021$ & Itajuba & 18 May 02 & 05:09 - 07:42 & 2.6 \\
\hline jgp0217 & Tenerife & 19 May 02 & $01: 33-05: 02$ & 3.5 \\
\hline lna-023 & Itajuba & 19 May 02 & $05: 25-07: 42$ & 2.3 \\
\hline mdr201 & McDonald & 19 May 02 & $08: 52-09: 28$ & 0.6 \\
\hline $\operatorname{lna}-027$ & Itajuba & 20 May 02 & $05: 19-07: 18$ & 2.0 \\
\hline mdr202 & McDonald & 20 May 02 & $05: 33-08: 25$ & 2.9 \\
\hline mdr202b & McDonald & 20 May 02 & $09: 09-09: 26$ & 0.3 \\
\hline mdr203 & McDonald & 21 May 02 & $03: 46-08: 22$ & 4.6 \\
\hline mdr203b & McDonald & 21 May 02 & $09: 01-09: 26$ & 0.4 \\
\hline
\end{tabular}


The times are all relative to the beginning of the overlap, and the time shifts are added to the data set that starts later. Most of the necessary offsets are of the order of what is expected from a preliminary data reduction of such an inhomogenous overall data set; the shift between ra393 and lna-006 however amounts to a much larger value which causes a phase shift close to one for the main pulsation period. It is also of the order of the barycentric correction, which might explain the origin of this discrepancy.

The fluxes are given as modulation intensities, with small offsets to separate overlapping data sets and larger offsets to separate unrelated pairs of data. A comparison of the same fluxes from different measurements shows good agreement down to very small features.

In conclusion, although the pairs of data sets just presented are not necessarily representative for the whole data, the well-known need to very carefully extract the time stamps for the data points in the final reduction has become obvious again, but the flux values of the light curve probably already look very similar to those that the final reduction will yield in the end.

The next subject of importance is the frequency resolution that can be achieved for a temporal spectrum of the data set. A complete separation of two frequencies is possible if they are separated by more than $1.5 / T$ which is equal to $1.05 \mu \mathrm{Hz}$ for the WET data ${ }^{2}$. Since the confidence in the detection of a frequency enormously raises if it is independently found in both parts of a data set split in two halves, so

Table 4. Overlapping data sets and relative time shifts.

\begin{tabular}{llrrl}
\hline Run1 & Run2 & Overlap & Shift & Plot \\
\hline dod059 & jes0202 & $0.19 \mathrm{~h}$ & & \\
B0021 & korea006 & $1.51 \mathrm{~h}$ & & yes \\
ra393 & lna-006 & $2.11 \mathrm{~h}$ & $443 \mathrm{~s}$ & yes \\
jes0208 & lna-008 & $2.39 \mathrm{~h}$ & $37 \mathrm{~s}$ & yes \\
jkt-002 & mp-001 & $1.33 \mathrm{~h}$ & $-5 \mathrm{~s}$ & yes \\
jkt-002 & loi-002a & $0.29 \mathrm{~h}$ & & \\
mp-001 & loi-002a,b & $1.30 \mathrm{~h}$ & $12 \mathrm{~s}$ & yes \\
loi-004 & jkt-004 & $1.78 \mathrm{~h}$ & $6 \mathrm{~s}$ & yes \\
a0236 & pab-0197 & $0.16 \mathrm{~h}$ & & \\
lna-018 & a0238 & $0.12 \mathrm{~h}$ & & \\
loi-005 & lna-021 & $0.14 \mathrm{~h}$ & & \\
lna-027 & mdr202 & $1.75 \mathrm{~h}$ & & yes \\
\hline
\end{tabular}

\footnotetext{
${ }^{2} T$ denotes the time base and amounts to $1425600 \mathrm{~s}$ for the WET data.
} 


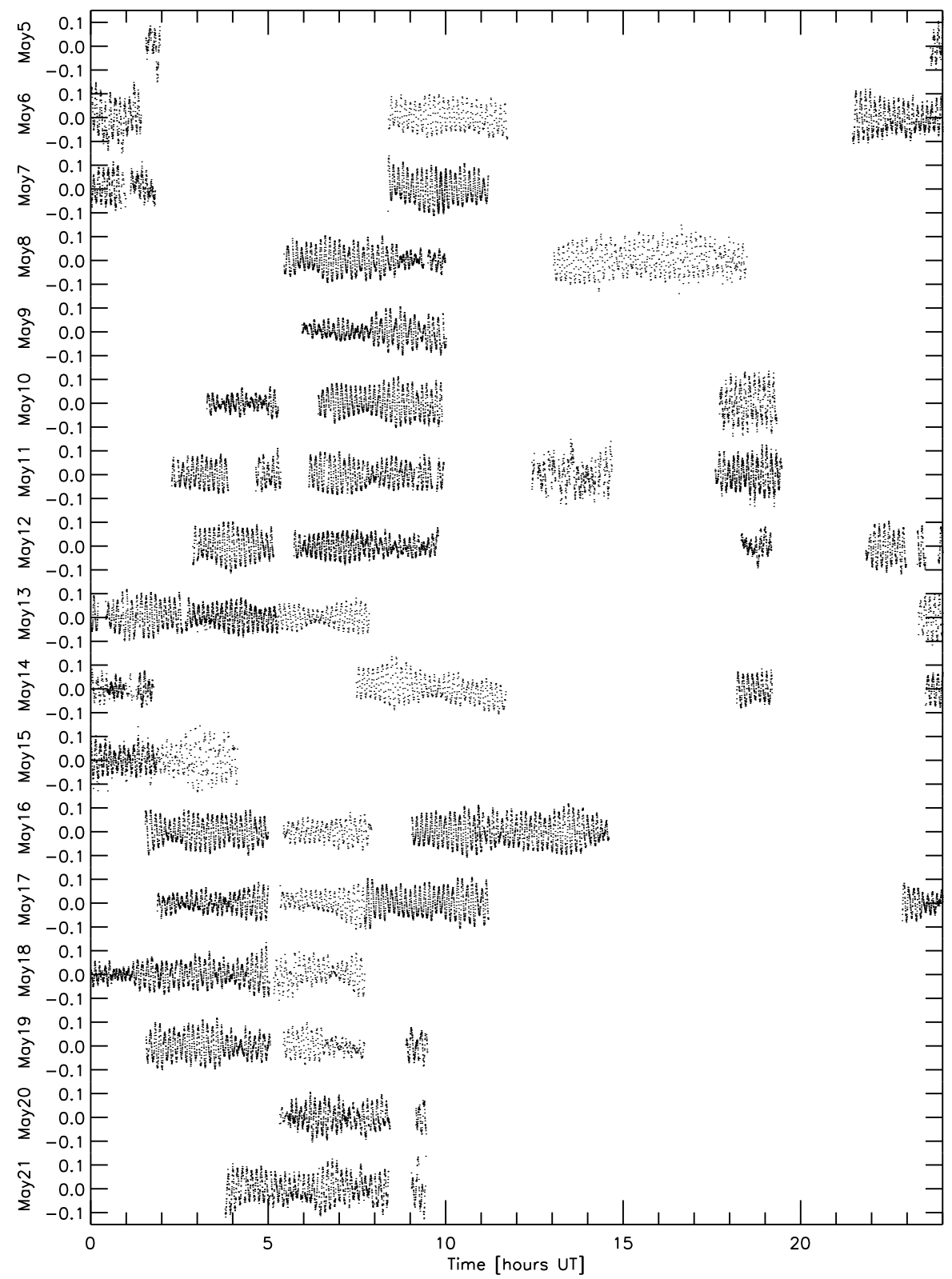

Fig. 1. Overview of the data obtained by the Whole Earth Telescope. 


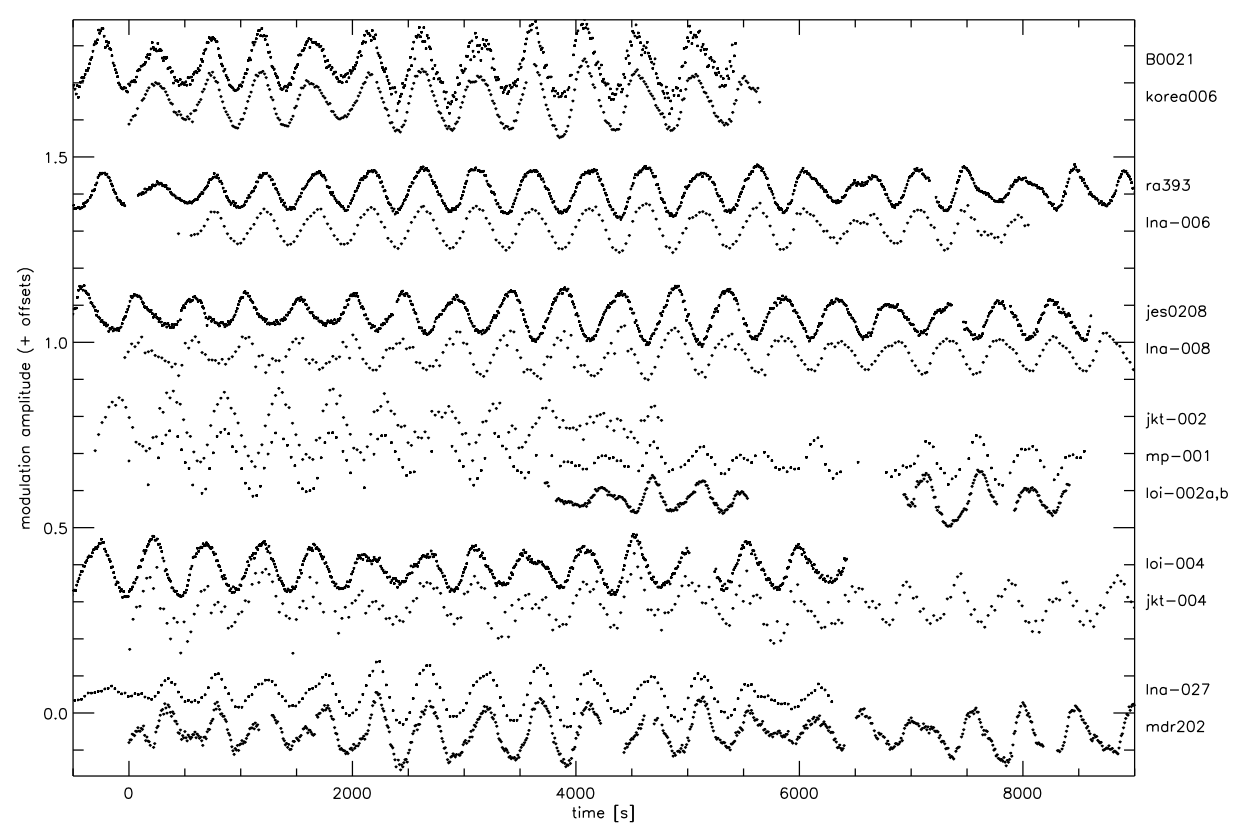

Fig. 2. Overlapping data; see text for explanation.

that one certainly wants to use this method, the resolution degrades to above $2 \mu \mathrm{Hz}$ for the individual halves of data. This is, for comparison, only marginally above the frequency separation of $\Delta f=1.6 \mu \mathrm{Hz}$ between the two large amplitude, closely spaced frequencies $f_{2}$ and $f_{3}$ in Kilkenny et al. (1999), and below the smallest separations between individual lower amplitude frequencies found in that paper. Since the latest WET run alone already exceeds the time base of their multi-site campaign, and together with the MSST photometric data, the nominal resolution of $0.58 \mu \mathrm{Hz}$ will be smaller by a factor of two as compared with their main campaign, it can nevertheless be assumed that the frequencies found by Kilkenny et al. will also be recovered in the new data provided they are still present in the stellar pulsation spectrum.

However, another prerequisite for the successful disentanglement of such a temporal spectrum is a high filling factor for the light curve and a well behaved window function to avoid confusion in the search for the position of frequencies by large aliases. The window functions for the full WET data as well as for its first and second halves separately are shown in Fig. 3 (in pseudo-amplitude units). The dominant features are in all cases still the daily aliases, which vary in height from 0.47 times the height of the main peak in the full 


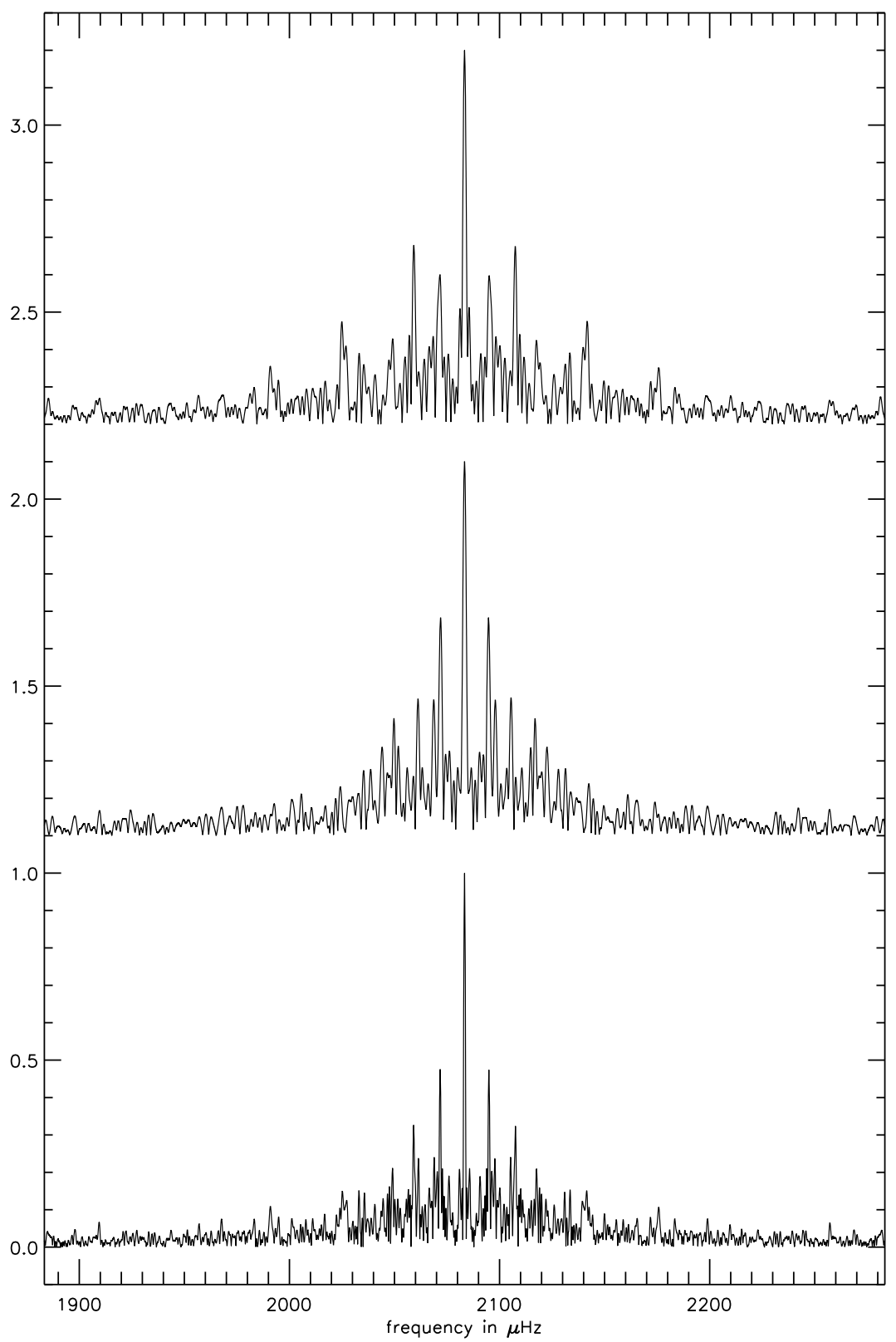

Fig. 3. Window functions for a sinusoid at $2083 \mu \mathrm{Hz}$ (corresponding to the main period), from top to bottom: first half, second half, full run. All functions are given in pseudo-amplitude units (square root of power spectral density), and are normalized to unity at the maximum peak height. The upper two are plotted with offsets. 
data set and its first half, to up to 0.58 times the height of the main peak for the second half of the divided data set. The filling factor for the combined WET and MSST photometry is higher than for the WET data alone, and the regular daily gaps are partly filled in by later MSST observations. A further improvement of the window function can therefore be expected for the combined light curve.

\section{SUMMARY}

As for the other MSST sub-projects, the considerable amount of data gathered during the WET campaign now needs to be subjected to a careful final reduction. From what has been said above, the very satisfactory data quality provides an excellent basis to start with. The next steps will then be to combine the WET data with the MSST photometry, and to do the frequency analysis on the joined data. A list of periods and amplitudes will constitute the intermediate data product that can then be brought together with data products from the spectroscopic MSST data, at which point the process will become increasingly exciting.

In sum, the Multi-Site Spectroscopic Telescope campaign, backed by the Whole Earth Telescope, has succeeded in accumulating an extraordinary and unique data set. It has a great potential to actually achieve its goal of arriving at a mode identification for the pulsational frequencies of PG 1605+072, and the scientific implications of the results are certainly eagerly awaited by many of us.

\section{REFERENCES}

Charpinet S. 2001, AN, 322, 387

Falter S. 2001, Diploma Thesis, Universität Erlangen-Nürnberg

Falter S., Heber U., Dreizler S., Schuh S.L., Cordes O. 2003, in 13th European Workshop on White Dwarfs, eds. R. Silvotti \& D. de Martino, Kluwer Academic Publishers, NATO Science Series, in press

Green R. F., Schmidt M., Liebert J. 1986, ApJS, 61, 305

Heber U., Reid N., Werner K. 1999, A\&A, 348, L25

Heber U. et al. 2003, in 13th European Workshop on White Dwarfs, eds. R. Silvotti \& D. de Martino, Kluwer Academic Publishers, NATO Science Series, in press

Jeffery C. S., Pollacco D. L. 2000, MNRAS, 318, 974

Kawaler S.D. 1999, in 11th European Workshop on White Dwarfs, eds. J.-E. Solheim \& E. Meištas, ASP Conference Series, 169, 158 
Kilkenny D., Heber U., Drilling J. S. 1988, SAAO Circ., No. 12

Kilkenny D., Koen C., O'Donoghue D., van Wyk F., Larson K. A., Shobbrook R., Sullivan D., Burleigh M., Dobbie P. D., Kawaler S. D. 1999, MNRAS, 303, 525

Koen C., O'Donoghue D., Kilkenny D., Lynas-Gray A. E., Marang F., van Wyk F. 1998, MNRAS, 296, 317

O'Toole S. J., Bedding T. R., Kjeldsen H., Teixeira C., Roberts G., van Wyk F., Kilkenny D., D'Cruz N., Baldry I. K. 2000, ApJ, 537, L53

O'Toole S. J., Bedding T. R., Kjeldsen H., Dall T. H., Stello D. 2002, MNRAS, 334, 471

Riddle R. 2003, in The 6th Whole Earth Telescope Workshop (these proceedings), eds. J.-E. Solheim \& E. G. Meištas, Baltic Astronomy, 12, 183

Woolf V. M., Jeffery C. S., Pollaco D. L. 2002, MNRAS, 329, 497 